\title{
Ergebnisse der Foraminiferenanalyse zur Quartärstratigraphie in Skandinavien *)
}

\author{
Karen Luise Knudsen \& Rolf Wilhelm Feyling-Hanssen *) \\ Foraminifera, Quaternary, stratigraphy, micropalaeontology, marine sediments \\ Skandinavia
}

\begin{abstract}
$\mathrm{Z}$ us a m menfassung: In Skandinavien sind zahlreiche quartärstratigraphische Untersuchungen mit Hilfe fossiler Foraminiferen durchgeführt worden. Im folgenden wird eine Übersicht über die foraminiferenstratigraphischen Untersuchungen und deren Resultate in Dänemark, Norwegen und Schweden gegeben.
\end{abstract}

[Contribution to Quaternary Stratigraphy in Scandinavia by Foraminiferal analyses].

A bstract: A considerable number of papers on fossil foraminifera and their application to Quaternary stratigraphy has appeared in Scandinavia. In the following these investigations and their results are reviewed and exhaustive references given.

\section{Einleitung}

Seit mehr als zweihundert Jahren kennt man fossile Foraminiferen in marinen Ab-

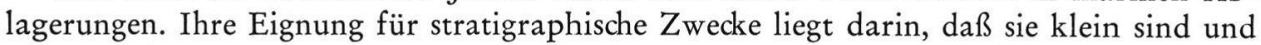
oft in großen Mengen im Sediment vorkommen. Untersuchungen von rezenten Foraminiferen haben gezeigt, daß die verschiedenen Arten abhängig sind von bestimmten ökologischen Verhältnissen, z. B. von der Temperatur, der Tiefe und der Salinität. Das bedeutet, daß man Foraminiferen auch innerhalb des Quartärs in der Stratigraphie anwenden kann, da Klimaschwankungen sich in marinen Sedimenten dieser Periode aufgrund von Änderungen der Foraminiferenfauna abzeichnen.

Es ist bekannt, daß wesentliche quartärstratigraphische Resultate durch Untersuchungen der planktonischen Foraminiferen-Gesellschaften in Bohrkernen aus der Tiefsee erzielt wurden. Aber auch Untersuchungen von Foraminiferen in marinen quartären Ablagerungen von Tagesaufschlüssen und Bohrungen auf der Landoberfläche haben bedeutende Resultate gezeitigt. In solchen Untersuchungen spielen die benthonischen Foraminiferen normalerweise eine Hauptrolle. Im allgemeinen benutzt man in der Foraminiferenstratigraphie quantitative Analysen der Foraminiferengesellschaften, so z. B. für die paläoökologische Auswertung die zahlenmäßige Repräsentation der einzelnen Arten in den Proben und verschiedene andere einfache ökologische Maße.

In Skandinavien hat man zahlreiche quartärstratigraphische Untersuchungen durchgeführt. Die Resultate findet man verstreut in Monographien und Artikeln in vielen verschiedenen Zeitschriften. In den meisten Fällen geht aus dem Titel direkt hervor, daß es sich um quartärstratigraphische Abhandlungen handelt, aber in einigen Fällen ist der stratigraphische Inhalt verborgen. Im folgenden haben wir versucht, eine Zusammenfassung

*) Nach einem am 21. 9. 1976 in Aarhus auf der DEUQUA-Skandinavien-Exkursion gehaltenen Vortrag.

*) Anschrift der Verfasser: Dr. K. L. K n u d s e n und Dr. R. W. F e y ling - H a n s s e n, Afdelingen for Mikropalaeontologi, Aarhus Universitet, DK-8000 Aarhus C, Danmark. 
über die foraminiferenstratigraphischen Untersuchungen und deren Resultate in Dänemark, Norwegen und Schweden zusammenzustellen. Die Ergebnisse sind fragmentarisch und unzusammenhängend, weil die Untersuchungen in den Randgebieten des NordseeBeckens durchgeführt wurden. Das Vorhandensein oder Fehlen von stratigraphischen Einheiten ist abhängig von Transgressionen und Regressionen, die diese Randgebiete berührt haben. Zukünftige Untersuchungen sollten in größerem Maße Material vom zentralen Teil des Beckens berücksichtigen, wo wahrscheinlich eine zusammenhängende quartärstratigraphische Dokumentation vorliegen wird.

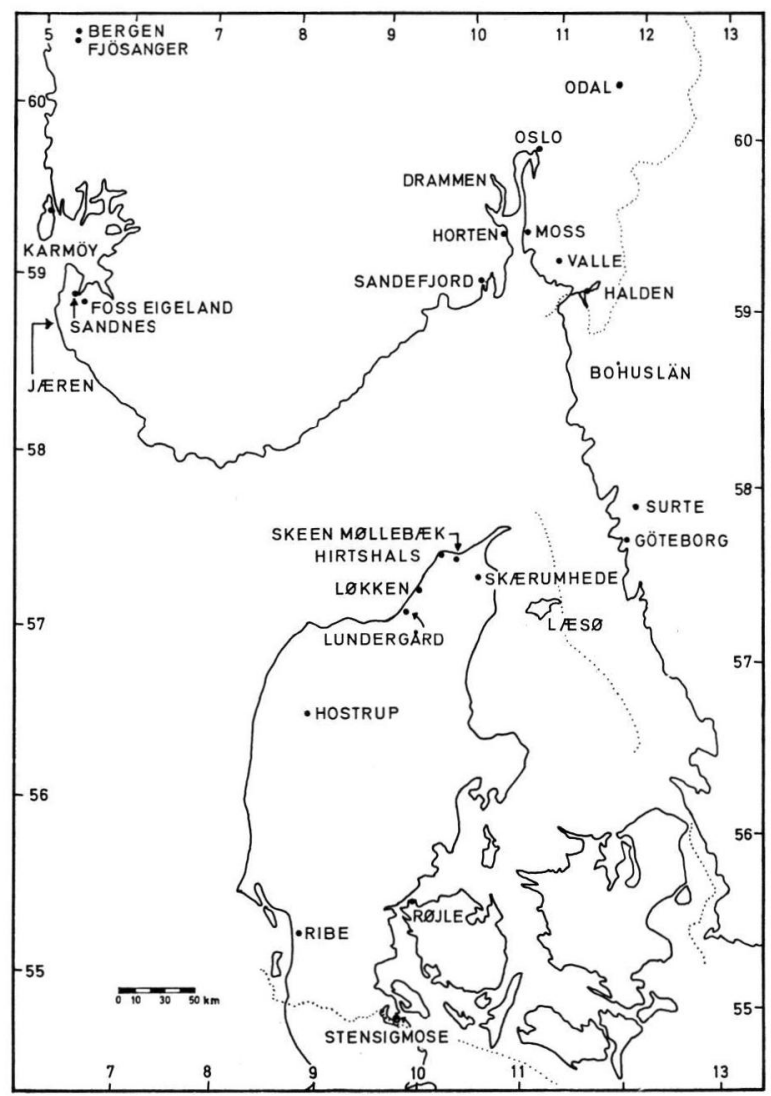

Abb. 1: Erwähnte Fundorte von Foraminiferen in Skandinavien.

\section{Spätweichsel-Kaltzeit und die Flandrischen Ablagerungen im Oslofjord-Gebiet, Norwegen}

Die isostatische Landhebung nach der letzten Eiszeit führte zu einer bedeutungsvollen negativen Strandverschiebung im Oslofjord-Gebiet. Vor zirka 9500 Jahren lag die Strandlinie bei Oslo $221 \mathrm{~m}$ höher als heute. Durch diese Hebung wurden größere marine Bereiche zu Festland. Seit mehr als einem Jahrhundert sind sie Gegenstand geologischer Untersuchungen. Hier ist es möglich, bestimmte marine Horizonte mit bestimmten Stadien des Eisrand-Rückzuges zu korrelieren, da man im Gebiet Gletscher-Randmoränen findet. Die markanteste Randmoräne ist die „Ra“-Moräne. Sie erstreckt sich von Halden über 
Sarpsborg und Moss nach Horten und Sandefjord-Larvik. Der Rand des skandinavischen Inlandeises befand sich vor $10300-10000$ Jahren in dieser Position.

SARs \& KJerulf (1861) gliederten die marinen Tone und Molluskenbänke im Oslofjord in einen glazialen und einen postglazialen Abschnitt. Im glazialen Abschnitt identifizierte SARS (1865) 6 Foraminiferenarten und im postglazialen 18. Im letztgenannten fand er Ammonia beccarii als Leitfossil.

Crosskey \& Robertson (1868) registrierten 48 Arten von Foraminiferen in Proben von quartären Ablagerungen in Oslo und Umgebung. KI£R publizierte (1900) eine Liste von Foraminiferenarten, die er in quartären Proben fand, die SARS und MüNSTER eingesammelt hatten. Aufgrund von fossilen Mollusken teilte BrøgGer (1901) die marinen Tone und gröberen Flachwasserablagerungen in eine Anzahl Zonen und Schillhorizonte ein. In dieser Arbeit publizierte KIÆR Listen von Foraminiferen, die er in einigen von BRøGGER's Molluskenzonen gefunden hatte. Es sei erwähnt, daß KIÆR (1908) auch Foraminiferen von quartären Ablagerungen von Tromsø in Nord-Norwegen und MoNSEN (1934) von quartären Ablagerungen in Nordre Helgeland beschrieben haben.

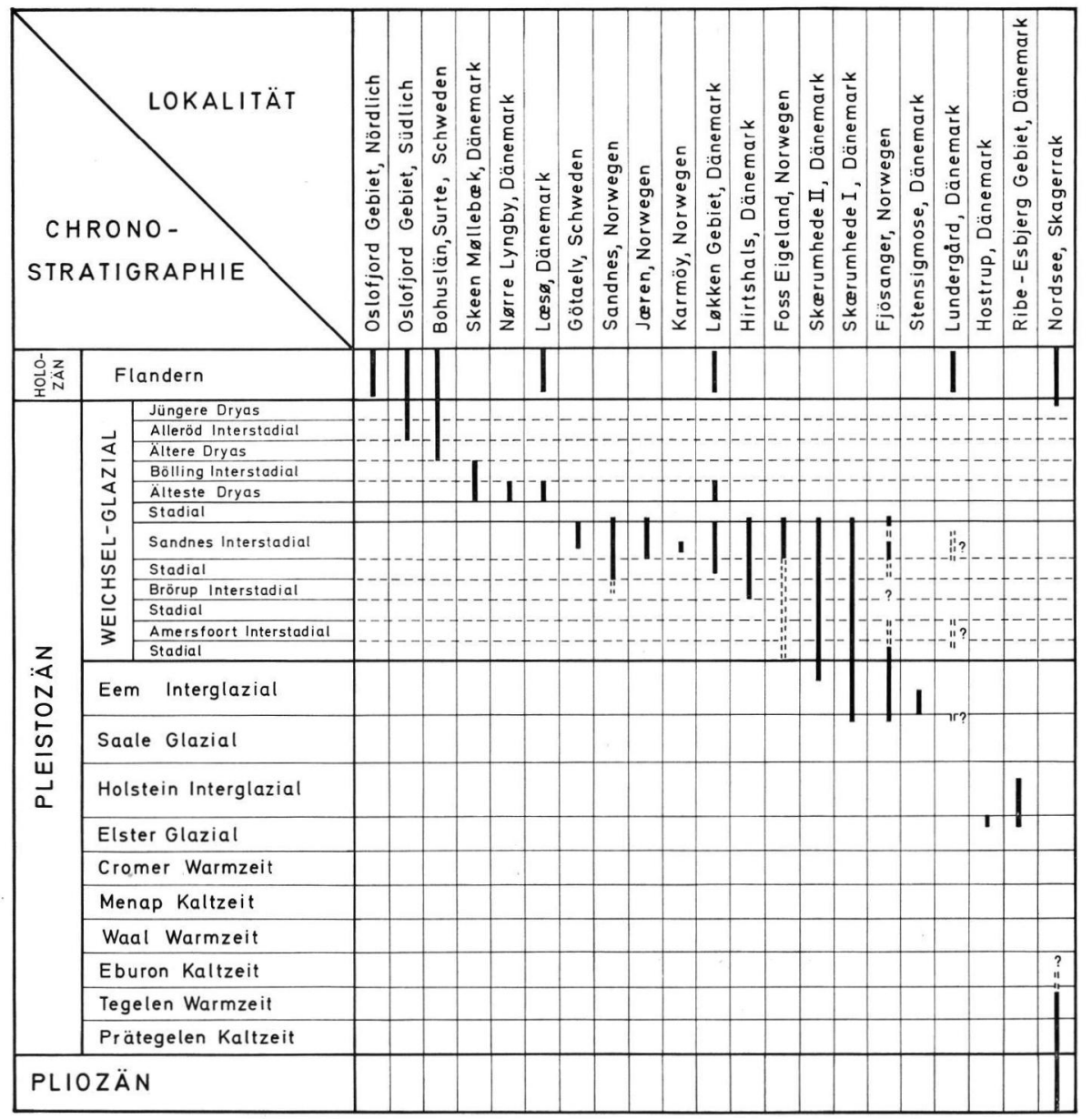

Abb. 2: Stratigraphische Einordnung der marinen Quartärablagerungen eines Teils der skandinavischen Fundorte, von denen Foraminiferenfaunen beschrieben wurden. 
Feyling-Hanssen (1954a, 1955) führte quantitative Untersuchungen des Foraminifereninhaltes einiger Tonproben durch, die BRøGGER im Oslo-Gebiet gesammelt hatte und präsentierte die prozentuale Zusammensetzung der Foraminiferengesellschaften, die Brøgger's Yoldia-Ton, Arca-Ton und Isocardia-Ton entsprachen. Auf diese Weise konnte ein Isocardia-Ton und ein Arca-Ton ausschließlich mit Hilfe des Foraminifereninhaltes identifiziert werden (FeyLING-HansSen 1954b). Die Foraminifere Elphidium excavatum forma clavata wurde in marinen Ablagerungen im Norden bei Odal gefunden (FEYLINGHANSSEN $1954 \mathrm{c}$ ).

FEYLING-HANSSEN (1957) gliederte marine Quartärablagerungen in Oslo und Umgebung in 5 Foraminiferenzonen: C, D, E, F und G. Alle gehören dem Holozän an. Die Zonen $\mathrm{C}$ und $\mathrm{D}$ haben spätglazialen Charakter, die darüberliegenden Zonen E-G postglazialen Charakter. Die Zonen D-G wurden bei Drammen wiedergefunden (RISDAL 1962). Feyling-HANSSEN (1964a) untersuchte den quantitativen Foraminifereninhalt in 2500 Kernproben von 130 Bohrungen in den marinen Quartärablagerungen des gesamten Oslo-Gebietes von Odal im Norden bis Halden, Sandefjord und Larvik im Süden. Er gliederte hierdurch die Ablagerungen in 7 Zonen mit den Bezeichnungen: A (älteste) - G (jüngste). Die Zone A wurde in drei Subzonen unterteilt: A-unten, A-mittel, A-oben; von diesen gehören $A_{u}$ zur "Jüngeren Dryas“ oder zum „Oberen Allerød“ ( ${ }^{14} \mathrm{C}-$ Datierung $11200 \pm 200$ B.P.). $A_{m}$ wurde in die "Jüngere Dryas“ gestellt ( ${ }^{14} \mathrm{C}$-Datierung $11000 \pm 225$ B.P.) und die Subzone $A_{o}$ ergab „präboreales" Alter (14C-Datierung 9950 \pm 300 B.P.). Die Pleistozän/Holozän-Grenze wurde zwischen den Subzonen $A_{m}$ und $A_{o}$ gezogen (Feyling-HansSEn 1972).

Eine ${ }^{14} \mathrm{C}$-Datierung einer Probe aus der Zone B ergab ein Alter von $9750 \pm 250$ B.P. („Präboreal“). Die Zone E gehört ins „Boreal“ (14C-Datierung $9100 \pm 180$ B.P.) und die Zone F ins "Atlantikum" und "Subboreal" ("14C-Datierung $6570 \pm 150$ B.P.). Die Zone G hat hauptsächlich "subatlantisches Alter" (14C-Datierung $2050 \pm 150$ B.P. und $980 \pm 100$ B.P.). Sie tritt auch als Flachwasserfazies der Zone F auf. RiSDAL (1964) verglich diese Zonen mit rezenten Foraminiferen im Oslofjord.

\section{Das Spätglazial und die Flandrischen Ablagerungen im Skagerrak}

LANGE (1956) beschrieb postglaziale und einige spätglaziale Gesellschaften von fossilen Foraminiferen aus kurzen Bohrkernen im Skagerrak. KIHLE (1971) fand postglaziale Vorkommen in 5 kurzen Bohrkernen von der "Norwegischen Rinne“ südlich von Mandal, während FÄLT (1977) unter den postglazialen Ablagerungen in zwei kurzen Bohrkernen in der "Tiefen Rinne" zwischen Skagen und der Schwedischen Westküste auch spätglaziale Ablagerungen fand.

\section{Das Spätglazial und die Flandrischen Ablagerungen in Bohuslän und Göteborg, Schweden}

Munthe beschrieb 1896 fossile Foraminiferen im Yoldia-Ton von Mälardalen und von der Insel Tjörn im Bohuslän-Gebiet und 1901 von der Ebene von Västergötland. HEssland (1943) registrierte Foraminiferen in spätglazialen und postglazialen marinen Schalenablagerungen im Nord-Bohuslän und stellte unter anderen ein Diagramm über die Einwanderungsintensität der Foraminiferen von ca. 10000 B.C. bis ca. 5000 B.C. auf. In den älteren Sedimenten wurden 32 Arten gefunden. Bis ca. 7500 B.C. war die Zahl der Arten auf 58 angestiegen und 5000 B.C. lag sie bei 75.

Brotzen beschrieb, in der bereits genannten Arbeit von Hessland (1943), zwei neue Arten aus dem spätglazialen Mergelton von Halland, nämlich Elpbidium asklundi und E. hallandense. Im Jahr 1951 benutzte BROTZEN die quantitative Foraminiferenanalyse 
für eine stratigraphische Untersuchung von zwei Bohrungen in spät- und postglazialen Ablagerungen bei Surte in der Nähe von Göteborg. Drei genau definierte Abschnitte konnten gefunden werden. Der älteste Abschnitt recht vermutlich bis in die „Altere Dryas“ zurück.

\section{Die Spätweichsel-Kaltzeit und die Flandrischen Ablagerungen in Nordjütland, Dänemark}

In Nordjütland liegen große Gebiete mit marinen spät- und postglazialen Ablagerungen über dem heutigen Meeresniveau, bedingt durch die isostatische Hebung des Gebietes nach der Weichsel-Vereisung. Die spätglazialen marinen Ablagerungen in Nordjütland sind ${ }^{14} \mathrm{C}$-datiert auf $14650 \pm 190$ bis $11950 \pm 190$ B.P. (Krog \& TAUber 1974; Krog, pers. Mitt.) („Älteste Dryas“ und „Bölling-Interstadial“). MADSEN (1895) registrierte erstmalig Foraminiferen aus diesen Ablagerungen. Später wurden die Faunen einiger Lokalitäten in diesem Gebiet detaillierter beschrieben, unter anderen von der Insel Læsø (MICHELSEN 1967) und von Løkken (KNUDSEN 1971). JøRGENSEN (1971) beschrieb Foraminiferen von einzelnen Proben von Nørre Lyngby und Skeen Møllebæk. Eine detailliertere Beschreibung der Fauna und ihrer Entwicklung während „Altester Dryaszeit“ und „Bølling “ von diesen beiden Lokalitäten wird von KNUDSEN ausgearbeitet.

Nach der Transgression in der „Altesten Dryaszeit“ und im „Bølling“ folgt eine Regression im Vendsyssel-Gebiet. In einem Zeitabschnitt ist die isostatische Hebung des Gebietes stärker als der eustatische Anstieg des Meeresspiegels; und erst im „Atlantikum“ gibt es wieder eine Transgression in diesem Gebiet. Die marinen postglazialen Ablagerungen, die danach in Nordjuitland abgelagert wurden, sind auf $8280 \pm 140$ bis $2440 \pm 100$ B.P. datiert (KROG \& TAUBER 1974). Foraminiferenfaunen aus diesen Schichten sind unter anderem beschrieben worden von der Insel Læsø (Michelsen 1967), Birkelse (Jørgensen 1971), Løkken (KNUDSEN 1971) und Lundergård (KNUDSEN 1973a).

\section{Das Interstadial der mittleren Weichsel-Kaltzeit bei Götaälv, Schweden}

BRotZEN (1961) publizierte eine Untersuchung einer $92 \mathrm{~m}$ tiefen Bohrung von Ingebäk im Götaelvs-Tal $12 \mathrm{~km}$ nördlich von Göteborg. Er fand im obersten Abschnitt der Bohrung einen $15 \mathrm{~m}$ mächtigen Postglazial-Ton mit Ammonia beccarii. Darunter folgten erst $20 \mathrm{~m}$ Spätglazial-Ton mit einer gemäßigten Fauna und dann Spätglazial-Ton mit einer „kalten“ Fauna mit Cassidulina crassa. Nach einer Diskontinuität mit groben Schottern folgten $11 \mathrm{~m}$ Interstadial-Ton mit Cassidulina crassa und darunter $19 \mathrm{~m}$ glaziofluviatiler Sand, der das Grundgebirge überlagert. Diese Sukzession wurde in Göteborg wiedergefunden. Radiokarbon-Datierungen (von ganzen Kernproben) des interstadialen Tones ergaben ein Alter von 26700 bis 29000 B.P. Auf Grund dessen definierte Brotzen das „Götaälv-Interstadial“, welches er mit dem „Paudorfer-Interstadial“ korrelierte.

\section{Interstadial und Stadial der mittleren Weichsel-Kaltzeit in Sandnes und auf Jæren, Norwegen}

Helland $(1879,1885)$ nahm an, daß während der „großen Eiszeit“ ein riesiger Skagerrak-Gletscher nach Westen in die Norwegische Rinne vorstieß und sich dann von Lista und Jæren aus (Südwestküste von Norwegen) nach Nordwesten und Norden vorschob. Dieser Gletscher transportierte marine Ablagerungen vom Boden des Skagerrak und erratische Blöcke vom Skagerrak, von Oslo und sogar aus dem Baltikum, auf das Tiefland von Jæren bis in eine Höhe von mehr als $200 \mathrm{~m}$ über dem jetzigen Meeresspiegel.

Die meisten späteren Forscher, die sich mit Jæren befaßt haben, griffen diesen Gedanken auf, weil man dadurch das Vorhandensein von marinen Fossilien und erratischen Blöcken aus einem entfernten Ursprungsgebiet hoch über der vermuteten marinen Grenze 
auf Jæren erklären konnte. Eine Ausnahme jedoch war Grimnes (1910), der den Mergelton auf Jæren nicht als eine sogenannte Skagerrakmoräne ansah, sondern als wirkliche marine Ablagerungen, ablagert dort, wo man sie heute findet, in einem Meeresniveau mehr als $200 \mathrm{~m}$ höher als das jetzige.

Mehrere Autoren faßten jedoch die mächtige tieferliegende Tonschicht in Sandnes, nördlich auf Jæren, als ein echtes marines Sediment auf.

Feyling-Hanssen (1964b) fand eine fossile Foraminiferenfauna in den Tonen bei Sandnes. Sie besteht hauptsächlich aus arktischen Arten, mit einigen borealen Arten. Gleichzeitig beschrieb er fossile Foraminiferen von Lokalitäten auf Jæren, u. a. von einer, die $175 \mathrm{~m}$ ü. NN und einer anderen, die $198 \mathrm{~m}$ ü. NN liegt. Diese Faunen konnten mit dem Sandnes-Ton korreliert werden. Er folgerte daraus: wenn der Sandnes-Ton marine Ablagerungen repräsentiert, dann sind die übrigen fossilführenden Ablagerungen auf Jæren ebenfalls marin. Die Annahme von GrimnEs von einer früheren ca. $250 \mathrm{~m}$ hoch liegenden Strandlinie auf Jæren muß daher richtig sein. Auch ANDERsen (1964) teilt diese Auffassung.

Als Resultat der Foraminiferenanalyse legte Feyling-Hanssen (1966) eine Zoneneinteilung von 1 bis 4 für eine 26,30 m tiefe Bohrung des Sandnes-Tones bei Gann, Sandnes, vor. Die oberste Zone 1, konnte mit dem „Paudorfer-Interstadial“ gleichgestellt werden.

Im Artikel „Eldre Yoldia ler på den norske Nordsø-kyst“ (FeYling-Hanssen 1970) wird auf die Gleichheit zwischen den Sandnes-Jæren-Ablagerungen und dem Ailteren Yoldia-Ton im Vendsyssel-Gebiet in Jütland aufmerksam gemacht und in der Arbeit "Weichselian interstadial foraminifera from the Sandnes-Jæren area" (FEYLING-HANSSEN 1971) wird das „Sandnes-Interstadial“ als ein längeres Weichsel-Interstadial aufgestellt. Hierunter gehören auch die Zonen 1 und 2 des Sandnes-Tones und der marine Ton in vielen Tagesaufschlüssen von Jæren. Radiokarbon-Datierungen dieser Ablagerungen (eingesandt von B. G. ANDERSEN) ergaben ein Alter von 28000 bis 42000 B.P. Die Foraminiferenfaunen der Lokalitäten Bø und Nygaard auf Karmøy, $65 \mathrm{~km}$ nördlich von Sandnes, wurden ebenfalls in dieses Interstadial gestellt. Eine ${ }^{14} \mathrm{C}$-Datierung ergab $34000 \pm$ 3000 B.P.

Die Zone 3 des Sandnes-Tones, die sich unterhalb der interstadialen Ablagerungen befindet, ist charakterisiert durch eine hocharktische Flachwasser-Foraminiferenfauna und repräsentiert somit ein vorausgehendes Stadial.

\section{Interstadial und Stadial der mittleren Weichsel-Kaltzeit bei Foss-Eigeland, Norwegen}

Bei Foss-Eigeland, $6 \mathrm{~km}$ südlich von Sandnes, findet man unter großblockiger Moräne der maximalen Weichsel-Vereisung marinen Ton mit einer Foraminiferenfauna der gleichen Zusammensetzung wie von der Zone 1 des Sandnes-Tones. Sie gehört demnach zum „Sandnes-Interstadial“ (FeYling-Hanssen 1966). Dieser Ton liegt bei Foss-Eigeland $70 \mathrm{~m}$ ü. NN und wird unterlagert von einer Moräne oder von periglazialen Ablagerungen, die einem kalten Intervall entsprechen, welches mit der marinen Zone 3 im Sandnes-Ton korreliert werden kann. Dieses kalte Intervall wurde „Foss-Eigeland-Stadial“ genannt (FEYLING-Hanssen 1974). Die darunterliegenden Sand- und Kiesablagerungen gehören in frühere Abschnitte der Weichsel-Eiszeit.

\section{Weichsel, Eem und Saale bei Fjøsanger, Norwegen}

Marine Ablagerungen, die ins Eem und in die Weichsel-Kaltzeit gehören, wurden von Fjøsanger südlich von Bergen in Norwegen beschrieben (MANGERud 1970, 1972). Das ma- 
rine Eem bei Fjøsanger ruht auf einer Moräne und ist von marinen Ablagerungen der Weichsel-Zeit überlagert. Hierüber folgt eine neue Moräne. Eine ${ }^{14} \mathrm{C}-\mathrm{Datierung}$ von einem Teil der marinen Serie, die zur Weichsel-Zeit gerechnet wird, ergab ein Alter von ca. 46000 B.P.

Eine Beschreibung der Foraminiferenfaunen des marinen Eem und der Weichsel-Zeit von Fjøsanger wird zur Zeit von Hans Petter S e j r u p in Bergen durchgeführt.

\section{Weichsel, Eem und Saale in Dänemark}

In einer Untersuchung der Foraminiferenfaunen vom Küstenprofil bei Hirtshals in Vendsyssel hat ANDERsen (1971) den Ailteren Yoldia-Ton in 7 Faunenzonen gegliedert, die Zonen A-F. Die ökologische Entwicklung, die sich in den obersten 3 Zonen der Faunen von Hirtshals abzeichnet, entspricht den Entwicklungen in den Zonen 1-3 in Sandnes (Feyling-Hanssen 1966, 1971), und die Zonen A und B in Hirtshals gehören ins „Sandnes-Interstadial“. Die beiden ältesten Zonen in Hirtshals, Zone E und F, entsprechen einer milden Periode der Weichsel-Kaltzeit. Sie können möglicherweise mit dem „Brørup-Interstadial“ korreliert werden. Zwei ${ }^{14} \mathrm{C}$-Datierungen aus der Zone E in Hirtshals ergaben ein Alter von mehr als 35000 B.P.

Andersen (1971) hat die 7 Zonen des Alteren Yoldia-Tons von Hirtshals mit den obersten $30 \mathrm{~m}$ der marinen Schichtfolge und dem untersten Teil der glaziofluviatilen Ablagerungen der Skærumhede-I-Bohrung korreliert. Diese enthalten eine ca. $123 \mathrm{~m}$ mächtige marine Schichtenfolge, die auf ca. $20 \mathrm{~m}$ Moräne und glaziofluviatilen Ablagerungen ruht. Sie wird überlagert von etwa $57 \mathrm{~m}$ glaziofluviatilem Material. Auf Grund des Molluskeninhaltes dieser marinen Ablagerung hat JESSEN sie in 3 Zonen gegliedert (JESSEN et al. 1910). Zuunterst kommt die Turritella terebra-Zone vor. Sie ist ca. $74 \mathrm{~m}$ mächtig und enthält eine boreal bis boreo-lusitanische Molluskenfauna. Danach folgt eine etwa $8 \mathrm{~m}$ mächtige Abra nitida-Zone mit einer boreo-arktischen Fauna und zuoberst folgt die Portlandia arctica-Zone, die $41 \mathrm{~m}$ mächtig ist und zum Teil arktische, zum Teil boreo-arktische Mollusken enthält. Foraminiferen aus der Skærumhede-I-Bohrung wurden von AxEL NøRVANG untersucht; seine Resultate sind nicht publiziert, aber sein unvollendetes Manuskript und das Probenmaterial liegen vor.

Das Alter der marinen Skærumhede-Serie ist lange Zeit diskutiert worden. Um genaeuere Informationen über diese Ablagerungen zu erhalten, wurde von 1971 bis 1972 die Skærumhede-II-Bohrung niedergebracht (durchgeführt von Danmarks Geologiske Undersøgelse). Sie liegt nur wenige Meter von der alten Bohrung entfernt. Die Foraminiferenfaunen in den marinen Schichten der Skærumhede-II-Bohrung wurden von KoNRADI \& KNUDSEN (1974, in BAHNSON et al. 1974) und von KNUDSEN (im Druck a) beschrieben. Auf Grund der Foraminiferen kann man diese Schichtfolge in 9 Faunenzonen gliedern. Die untersten 2 Zonen entsprechen jeweils dem obersten Teil der Turritella terebra-Zone und der gesamten Abra nitida-Zone, die in den obersten Teil des Eem gestellt werden. Danach folgen 7 Faunenzonen, die durch ihre Foraminiferen-Schwankungen 3 Stadialen und 3 Interstadialen entsprechen. Diese Zonen wurden mit der Portlandia arctica-Zone der Skærumhede-I-Bohrung korreliert und werden zur unteren und mittleren Weichsel gerechnet. Die Interstadiale können möglicherweise mit den Interstadialen "Amersfoort", „Brörup" und „Sandnes" korreliert werden. Hieraus ergibt sich wahrscheinlich eine kontinuierliche Sedimentation im Vendsyssel-Gebiet während der Eem-Warmzeit und der frühen und mittleren Weichsel-Kaltzeit bis zum Hauptvorstoß des Weichsel-Eises, der der etwa vor $18000-20000$ Jahren stattgefunden hat.

Das spärliche Foraminiferenmaterial, das aus der Skærumhede-I-Bohrung vorliegt, zeigt, daß unterhalb der Ablagerungen des untersten Teils der Turritella terebra-Zone 
wiederum kältere marin-klimatische Verhältnisse geherrscht haben, da man hier eine Dominanz von arktischen Arten findet (Feyling-Hanssen et al. 1971). In der marinen Skærumhede-Serie scheint somit das gesamte „Eem-Interglazial“ vorhanden zu sein. Die marine Schichtenfolge ruht auf einer etwa $20 \mathrm{~m}$ mächtigen Moräne, von der man annimmt, daß sie aus der Saale-Eiszeit stammt (JESSEN et al. 1910).

Die Portlandia arctica-Zone wurde bereits von Jessen et al. (1910) mit dem Alteren Yoldia-Ton korreliert, den man in einer Reihe von Tagesaufschlüssen im nördlichen Vendsyssel-Gebiet in Jütland findet. Der Foraminifereninhalt von Proben aus dem Alteren Yoldia-Ton in einigen von diesen Tagesaufschlüssen wurde zuerst von MAdSEN (1895) beschrieben. Später wurden die Faunen vom Küstenprofil bei Hirtshals, wie bereits erwähnt, von Andersen (1971) sehr detailliert beschrieben. Außerdem wurden die Foraminiferenfaunen im Alteren Yoldia-Ton später aus einer Reihe anderer Lokalitäten in diesem Gebiet beschrieben, unter anderem vom Küstenprofil nördlich von Løkken (KNUDSEN 1971) und von Frederikshavn (JørGENSEN 1971).

Von mehreren Lokalitäten in Nordseeland beschrieb Konradi (1974, in Petersen \& KONRADi 1974) umgelagerte Foraminiferen, die offensichtlich aus entsprechenden marinen Ablagerungen der Weichsel-Kaltzeit stammen. Außerdem beschrieb Buch (1974, in PeterSEN \& BUCH 1974) Foraminiferenfaunen in marinen quartären Ablagerungen bei Holmstrup in Nordseeland. Diese Faunen wurden mit Faunen in zwei der Zonen der Skærumhede-II-Bohrung korreliert. Die Ablagerungen scheinen demnach sowohl Interstadiale als auch Stadiale der Weichsel-Eiszeit zu repräsentieren.

Foraminiferenfaunen von marinen Schichten des Lundergård-Tones in Vendsyssel wurden von Knudsen $(1972,1973)$ untersucht. Das Alter dieser Schichten ist unsicher. Möglicherweise stammen sie aus der Saale-Eiszeit, oder sie können eine spezielle Fazies des Älteren Yoldia-Tones repräsentieren und somit aus der Weichsel-Eiszeit stammen (Abb. 2).

Marine Ablagerungen aus dem untersten Teil des Eem kennt man in den Tagesaufschlüssen vom Süden Jütlands und den benachbarten Inseln. Foraminiferen aus diesen Eem-Ablagerungen wurden erstmalig von Madsen (1895, 1900), Munthe (1898), AnderSON (1898) und HARder (1900) erwähnt. Später beschrieb KonRadi (1976) ForaminiferenFaunen aus Eem-Ablagerungen bei Stensigmose in SO-Jütland detaillierter. Außerdem publizierte KONRADI Foraminiferenfaunen in marinen Quartärablagerungen auf SO-Seeland (in Petersen \& Konradi 1974) und auf Westmøn (in Bertelsen, Konradi \& PeterSEN, 1976). Aufgrund dieser Faunen nimmt man an, daß diese Ablagerungen ins Eem gehören.

\section{Holstein und Elster in Dänemark}

Foraminiferen von marinen Holstein-Sedimenten bei Esbjerg wurden zuerst von MAdSEN (1895) beschrieben. Später beschrieb Buch (1955) Foraminiferenfaunen aus den marinen interglazialen Ablagerungen in einer Bohrung bei Inder Bjergum in der Nähe von Ribe. Die Bohrung enthielt sowohl eine untere Serie mit einer arktischen Fauna, die wahrscheinlich dem Ende der Elster-Eiszeit entspricht, als auch eine obere Serie mit einer borealen Fauna, die in das „Holstein-Interglazial“ gehört. Ablagerungen entsprechenden Alters kennt man im nordwestlichen Schleswig-Holstein, und Foraminiferen von hier wurden unter anderem von LANGE (1962) und Woszidlo (1962) beschrieben. In Dänemark sind marine Ablagerungen bei Røgle Klint auf NW-Fyn in das „Holstein-Interglazial“ gestellt worden. Eine Liste der Foraminiferen von dieser Lokalität ist von MADSEN (1895) publiziert worden. Später hat Woszidlo (1962) eine kurze Beschreibung der Fauna aus einer einzelnen Probe dieser Lokalität gegeben. 
Foraminiferenfaunen von marinen Quartärablagerungen bei Hostrup in Nordjütland wurden von KNUDSEN beschrieben (im Druck b). Diese Faunen sind mit Faunen im tieferen Teil der marinen Serie der Inder-Bjergum-Bohrung korreliert und gehören wahrscheinlich die Spät-Elster.

\section{Das älteste Quartär in Dänemark}

Marine quartäre Ablagerungen älter als Holstein und Spät-Elster sind in Skandinavien unbekannt. Man kennt sie jedoch aus Bohrungen in der Nordsee. Foraminiferenfaunen von den jüngsten Tertiär- und von den älteren Quartärablagerungen wurden im dänischen Teil der Nordsee von Buch (1972) gefunden. Der quartäre Teil dieser marinen Ablagerungen wurde in die "Prätegelen-Kaltzeit" und in Abschnitte der "Tegelen-Warmzeit“ gestellt. Der obere Teil des Profils enthält derart wenig Foraminiferen, daß sie nicht für eine Datierung verwendet werden können.

Für die Ưbersetzung möchten wir Dr. Walter L. Friedrich herzlich danken.

\section{Schriftenverzeichnis}

Andersen, A.-L. L. (1971): Foraminifera from the Older Yoldia Clay at Hirtshals: 159-184. In: Feyling-Hanssen, R. W., Jørgensen, J. A., Knudsen, K. L. \& Andersen, A.-L. L. Bull. geol. Soc. Denmark, 21 (2-3): 67-317; Kopenhagen.

Andersen, B. G. (1964): Har Jæren vært dekket av en Skagerak-bre? Er „Skagerak-morenen“ en marin leire? - Norges geol. Unders., 228: 5-11; Oslo.

Andersson, Fr. (1898): Über die Quartäre Lagerserie des Ristinge Klints auf Langeland. Eine biologisch-stratigrafische Studie. - Bull. Geol. Inst. Univ. Upsala, 3: 115-180; Uppsala.

Bahnson, H., Petersen, K. S., Konradi, P. B., \& Knudsen, K. L. (1974): Stratigraphy of Quaternary deposits in the Skærumhede II boring: lithology, molluscs and foraminifera. - Danm. geol. Unders., Årbog 1973: 27-62; Kopenhagen.

Bertelsen, A., Konradi, P. \& Petersen, K. S. (1976): Kvartære lagfølger og strukturer i Vestmøns klinter. - Dansk geol. Foren., Arsskrift 1976: 93—99; Kopenhagen.

Brotzen, F. (1943): In: Hessland, I.: Marine Schalenablagerungen Nord-Bohusläns. - Bull. Geol. Inst. Upsala, 31: 267-269; Uppsala.

- (1951): Bidrag till de svenska marine kvartäravlageringarnas stratigrafi. - Geol. Fören. Stockholm Förh., 73: 57-68; Stockholm.

- (1961): An Interstadial (radiocarbon dated) and the substages of the last glaciation in Sweden. - Geol. Fören. Stockholm Förh., 83: 144-150; Stockholm.

BrøgGer, W. C. (1901): Om de Senglaciale og Postglaciale Nivåforandringer i Kristianiafeltet (Molluskfaunaen). - Norges geol. Unders., 31: 731 S.; Oslo.

Buch, A. (1955): De marine interglaciale lag ved Inder Bjergum. Foraminiferfauna og stratigrafi. - Meddr dansk geol. Foren., 12 (6): 593-652; Kopenhagen.

- (1972): Undersøgelser over det marine Kvartær i Nordsøområdet. - Dansk geol. Foren., Årsskrift 1971: 86-90; Kopenhagen.

Crosskey, H. W., \& Robertson, D. (1868): Notes on the Posttertiary geology of Norway. Proc. Phil. Soc. Glasgow, 6: Glasgow.

Feyling-Hanssen, R. W. (1954 a): Late Pleistocene Foraminifera from the Oslofjord Area, Southest Norway. - Norsk geol. Tidsskr., 33: 109-152; Oslo.

- (1954 b): The stratigraphic position of the quick clay at Bekkelaget, Oslo. - Norsk geol. Tidsskr., 33: 185-196; Oslo.

- (1954 c): Elphidium clavatum Cushman from the Late-Glacial of Romerike, Norway. Norsk geol. Tiddsskr., 33: 79-83; Oslo.

- (1955): Dominating Foraminifera from the Late Pleistocene of the Oslofjord area, Southeast Norway. - Actes IV. Congr. Intern. Quaternaire, Rome-Pise 1: 348-355; Rom.

- (1957): Micropaleontology applied to soil mechanics in Norway. - Norges geol. Unders., 197 (Auch Norges geotekniske Inst., Publ. No. 20): 69 S.; Oslo.

- 1964 a): Foraminifera in Late Quaternary deposits from the Oslofjord area. - Norges geol. Unders., 225: 383 S.; Oslo. 
- (1964 b): Skagerakmorenen på Jæren. - Norsk geogr. Tidsskr., 19: 301-317; Oslo.

- (1966): Geologiske observasjoner i Sandnes-området. (Geological observations in the Sandnes area, Southwest Norway). - Norges geol. Unders., 242: 26-43; Oslo.

- (1970): झEldre Yoldialer på den norske Nordsø-kyst. — In: Feyling-Hanssen et al.: Mikropalæontologiske iagttagelser inden for det marine kvartær. - Dansk geol. Foren. Årsskrift 1969: 61-65; Kopenhagen.

- (1971): Weichselian interstadial Foraminifera from the Sandnes-Jæren area: 72-116. - In: Feyling-Hanssen, R. W., Jørgensen, J. A., Knudsen, K. L., \& Andersen, A.-L. L. - Bull. geol. Soc. Denmark, 21 (2-3): 67-317; Kopenhagen.

- (1972): The Pleistocene/Holocene boundary in marine deposits from the Oslofjord area. Boreas, 1 (3): 241-246; Oslo.

- (1974): The Weichselian section of Foss-Eigeland, Southwestern Norway. - Geol. Fören. Stockholm Förh., 96: 341-353; Stockholm.

Feyling-Hanssen, R. W., Jørgensen, J. A., Knudsen, K. L., \& Andersen, A.--L. L. (1970): Mikropalæontologiske iagttagelser inden for det marine Kvartær. - Meddr dansk geol. Foren., Årsskrift 1969: 61-70; Kopenhagen.

- (1971): Late Quaternary Foraminifera from Vendsyssel, Denmark and Sandnes, Norway. Bull. geol. Soc. Denmark, 21 (2-3): 67-317; Kopenhagen.

FÄLt, L.-M. (1977): Gränsen Pleistocen/Holocen i marina sediment utanför svenska västkusten. Chalmers Tekn. Högsk., Göteborgs Univ., Geol. Inst., Publ. A 14; Gothenburg.

Grimnes, A. (1910): Jæderens jordbund. — Norges geol. Unders., 52: 104 S.; Oslo.

Harder, P. (1900): En ny sønderjydsk lokalitet for marint diluvium. — Meddr dansk geol. Foren., 1 (6): 83-96; Kopenhagen.

Helland, A. (1879): Om Glaciationen af Shetland og Orkney. — Arch. f. Mat. og Naturv., 4; Kristiana.

- (1885): Om Jæderens løse Afleiringer. - Meddel. fra Den naturh. Foren. i Kristiania, 27-42, Kristiania.

Hessland, I. (1943): Marine Schalenablagerungen Nord-Bohusläns. - Bull. geol. Inst. Upsala, 31 : 348 S., Uppsala.

Jessen, A., Milthers, V., Nordmann, V., Hartz, N. \& Hesselbo, A. (1910): En boring gennem de kvartære Lag ved Skærumhede. - Danm. geol. Unders., (II), 25: 175 S.; Kopenhagen.

Jørgensen, J. A. (1971): The Quaternary of Vendsyssel: 117-129. — In: Ferling-Hanssen, R. W., Jørgensen, J. A., Knudsen, K. L., \& Andersen, A.-L. L. - Bull. geol. Soc. Denmark, 21 $(2-3): 67-317$; Kopenhagen.

KIHLE, R. (1971): Foraminifera in five sediment cores in a profile across the Norwegian Channel south of Mandal. - Norsk geol. Tidsskrift, 51: 261-286; Oslo.

KIÆR, H. (1900): Synopsis of the Norwegian Marine Thalamorphora. - Rep. Norw. Fishery- and Marine Invest., 1 (7); Kristiania.

- (1908): I. Om kvartærtidens marine aflejringer ved Tromsø. II. Fortegnelse over Tromsøsundets foraminiferer. - Tromsø Mus. Årsh., 25; Tromsø.

KNudSEN, K. L. (1971): Late Quaternary Foraminifera from the Løkken area: 130-158. - In: Feyling-Hanssen, R. W., Jørgensen, J. A., Knudsen, K. L., \& Andersen, A.-L. L. - Bull. geol. Soc. Denmark, 21 (2-3); 67-317; Kopenhagen.

- (1972): The Lundergård Clay and its Foraminifera, a new formation in the marine Quaternary of Denmark. - Boreas, 1 (4): 289-297; Oslo.

- (1973 a): Foraminifera from Postglacial deposits of the Lundergård area in Vendsyssel, Denmark. - Bull. geol. Soc. Denmark, 22: 255-282; Kopenhagen.

- (1973 b): The Lundergård Clay of Vendsyssel, Denmark, and its foraminifera. - Bull. geol. Soc. Denmark, 22: 155-192; Kopenhagen.

- (im Druck a): Foraminifer faunas in Weichselian stadial and interstadial deposits of the Skærumhede boring, Jutland, Denmark. - Maritime Sediments, Spec. Publ. No. 1, pt. B Paleoecology and Biostratigraphy; Halifax.

- (im Druck b): Foraminifera faunas of the Quaternary Hostrup Clay from northern Jutland, Denmark. - Boreas; Oslo.

KonRadi, P. B. (1976): Foraminifera in Eemian deposits at Stensigmose, southern Jutland. Danm. geol. Unders., (II), 105: 57 S.; Kopenhagen.

Konradi, P. B., \& Knudsen, K. L. (1974): Foraminifera in the Skærumhede II boring: 43-57. In: Bahnson, H., Petersen, K. S., Konradi, P. B., \& Knudsen, K. L. - Danm. geol. Unders., Årbog 1973: 27-62; Kopenhagen. 
Krog, H., \& Tauber, H. (1974): C-14 chronology of Late- and Postglacial marine deposits in North Jutland. - Danm. geol. Unders., Årbog 1973: 93-105; Kopenhagen.

LANGE, W. (1956): Grundproben aus Skagerrak und Kattegat, mikrofaunistisch und sedimentpetrographisch untersucht. - Meyniana, 5: 51-86; Kiel.

- (1962): Die Mikrofauna einiger Störmeer-Absätze Schleswig-Holsteins. - N. Jb. Geol. Paläont., Abh., 115 (2): 222-242; Stuttgart.

Madsen, V. (1895): Istidens foraminiferer i Danmark og Holsten. - Meddr dansk geol. Foren., 2, 229 S.; Kopenhagen.

- (1900): The pleistocene Foraminifera of Sleswick and Holstein. - Meddr dansk geol. Foren., 6: 45-56; Kopenhagen.

MANGERUd, J. (1970): Interglacial sediments at Fjøsanger, near Bergen with the first Eemian pollen-spectra from Norway. - Norsk geol. Tidsskr., 50: 167-181; Oslo.

- (1972): The Eemian Interglacial and the succession of glaciations during the Last Ice Age (Weichselian) in Southern Norway. - Ambio Special Rep., 2: 39-44; Oslo.

Michelsen, O. (1967): Foraminifera of the Late-Quaternary deposits of Læsø. - Meddr dansk geol. Foren., 17: 205-263; Kopenhagen.

Monsen, A. (1934): Meddelelser om nogen skjellforekomster fra nordre Helgeland. - Bergens Mus. Årb.; Bergen.

Munthe, H. (1896): Till frågan om den baltiska Yoldiamergelns fauna. - Geol. Fören. Stockholm Förh., 18; Stockholm.

- (1898): Studien über ältere Quartärablagerungen im Südbaltischen Gebiete. - Bull. geol. Inst. Univ. Upsala, 3: 27-114; Uppsala.

- (1901): Om faunan i Vestgötaslättens Yoldialera mellan Skara-Herrljunga och Venern. Geol. Fören. Stockholm Förh., 23; Stockholm.

Petersen, K. S., \& Buch, A. (1974): Dislocated tills with Paleogene and Pleistocene marine beds. Tectonics, Lithology, macro- and microfossils. - Danm. geol. Unders., Årbog 1973: 63-91; Kopenhagen.

Petersen, K. S., \& Konradi, P. B. (1974): Lithologisk og palæontologisk beskrivelse af profiler i Kvartæret på Sjælland. - Dansk geol. Foren., Årsskrift 1973: 47—56; Kopenhagen.

RisDAL, D. (1962): En undersøkelse av kvartære ökostratigrafiske soner i Drammen, på grunnlag av foraminiferer. - Norges geol. Unders., 215: 68-86; Oslo.

- (1964): Foraminiferfaunaens relasjon til dybdeforholdene i Oslofjorden, med en diskusjon av de senkvartære foraminifersoner. - Norges geol. Unders., 226: $142 \mathrm{~S}$. (The bathymetrical relation of Recent Foraminiferal faunas in the Oslo Fjord with a discussion of the Foraminiferal zones from Late Quaternary time); Oslo.

SARs, M. (1865): Om de i Norge forekommende Fossile Dyrelevninger fra Quartærperioden. Et Bidrag til vor Faunas Historie. - Universitetsprogram; Christiania.

SARs, M., \& KJERUlf, Th. (1861): Iagttagelser over den Postpliocene eller Glaciale Formation i en Del af det sydlige Norge. - Universitetsprogram for første halvår; Christiania.

Woszidlo, H. (1962): Foraminiferen und Ostracoden aus dem marinen Elster-Saale-Interglazial in Schleswig-Holstein. - Meyniana, 12: 65-96; Kiel. 